\title{
Pooling of lupus can "catch" early skin cancerous: "Rapid Progression in DNA Damage"
}

\begin{abstract}
Immunity brought many unconventional, yet meets wisdom. Any diseases can be detected by our self-examination, that can a best examination ever or other screening measures before the symptoms become serious. Most cases have detected and diagnosed when other symptoms develop or developing. There are more than 1000 types of autoimmune diseases including breast cancer, skin cancer, lung cancer, colon cancer, prostate cancer, and lymphoma. Symptoms vary depending on the type and strength. Immunity can natural transition that club of biomedical changes that seen in human body progress [1]. It can an early begin research topic that has been traditionally treated with force of attraction. Medically with a fairly low level of success and does not address the most significant result, which include depression, sign of fearness, poignant, linking and insomnia. Medicine has been shown in preliminary studies to address and treat specific symptoms of lupus. Some studies show a significant improvement in symptoms compared to that of family history; however these studies need to be reproduced on a larger scale to influence the medical community that has not familiar with therapy.
\end{abstract}

Keywords: Lupus - DNA; Insomnia - Immunity

Submitted Date: 05 December 2017; Accepted Date: 18 May 2018; Published Date: 22 May 2018

\section{Summary}

Immunity has colligative properties as these properties [2], which depend on the number of molecules and its release in order present in the human nature. The properties of immunity have independent on the nature to construct. It has dependent on nature through observation. Immunity ascends to the top post in his own. Human body has asset of liquid which arises from the intermolecular forces of attraction. A molecule in the interior of liquid has attracted equally in all direction by the similar molecule around it. At the force however a molecule has only partially surrounded by similar molecules. The forces on the side being counter balanced the surface molecule can pulled only by the inward the liquid. The liquid surfaces have, therefore under tension and tend to contract to the smallest possible area. This can only reason that body assume in spherical shapes. The border side of body forms between two immiscible phases have called body interface. Water exists separately in boundary interface. Human body has largely due to the intermolecular attractions which resist the flow of a liquid. So that it also dependent on the shape, size and mass of the liquid molecule. Water has a commonest example of liquid molecule that resists the flow of infection by external forces. The higher the force of a liquid, the greater the resistance to infection. It has the resistance to the flow of liquid under an applied stress. Body temperature rises when immunity breakdown in small fragment of degraded chemistry and reduce the viscosity of liquid and attachment of boundary infection to white blood cells [3]. Ape has an example poses a risk to naive autoimmune diseases.
Rahul Hajare*

Post Doc Fellow, Indian Council of Medical Research, New Delhi, India. *Author for correspondence:

E-mail: rahulhajare@rediffmail.com 
The coefficient of body force has the force per breath to require maintaining a body mass difference between two parallel situations. Lupus reduces the force required to produce a movable energy between two parallel planes of a body liquid each, water and blood. Flow of infection has reciprocal of the force coefficient of body force of unknown liquid may be determined by density of the known body liquid. When a liquid flows through the cells tube, the time required for the liquid to pass between two marks (X and $\mathrm{Y}$ ) has determined $[3,4]$. The time required for the liquid to flow has determined and compared modified with the time required for the reference liquid of known force. We already known example has water, lupus booster. Force can used as a quality tools for evaluation of flow properties of various infection forms such as solid cough, overweight, redness of eyes, continuous coughing, swelling of neck will vary person to person and past habits. Structure of human body depends upon the force and stability of food forms. Food derivatives like glucose, sucrose, black chocolate, fluidity are widely used in formation to increase immunity, vegetable and gelatinous etc. are commonly used as suspending agents in the body. In designing immunity operation such as fight to infection flows of infection through cells tubes the knowledge of force cost of water has helpful. Force can also be used to confirm the structure of body and constitutive life can be defined. Constitutive life of human depends upon the body gravity, weight of body and liquidity. The immunity of human body can be experiment in two steps, first has determination of density of water in body and determination of time of flow. Clean and clear body and transfer known quantity of water in cellular system can help to build immunity. Human body has all about the molecular weight of the known and unknown compound. Uniformity of body, otherwise weakness of diseases will vary from human to human [5,6]. Human body has two difference forces like, cohesive forces are intermolecular attraction between like molecule who fight known infection and eradicate through the skin and second has adhesive forces are attraction between unlike molecule who fight unknown infection and eradicate through the brain [6]. Vegetable oil and water exist separately as two phase and boundary has molecular weight of given compound called body texture interface. Body has made up of hydrophilic and hydrophobic molecule and when they are ground state, they are in their $\mathrm{pH}$ neutral and should be normal function. Lupus can see, when lower mark of any hydrophilic stop continuing by the time with unknown microorganism that begins the raises the temperature at which body interface are not in equilibrium at one atmospheric pressure [4]. This paper has created large discussion of molecular weight of food solute by elevated human body.

\section{Conclusion}

Immunity in brain, inter-immunity -blood may not be enough to obliterate immunity till the society remain patriarchal, a child's immunity can determined by his father's immunity. The immunity identity of the human gets eroded once they are unthoughful. The attempt should therefore to be fight patriarchy.

\section{Acknowledgment}

This study has been guided under the unparallel supervision and guidance of Renowned Scientist Respected Dr. Ramesh Paranjape, Retd, Director and Scientist 'G' National AIDS Research Institute India. I express my sincere gratitude towards Sir for motivation and being great knowledge source for this work. I seek continuous support for my research career.

\section{Executive summary}

Immunity brought many unconventional, yet meets wisdom. Any diseases can be detected by our self-examination, that can a best examination ever or other screening measures before the symptoms become serious. Most cases have detected and diagnosed when other symptoms develop or developing. There are more than 1000 types of autoimmune diseases including breast cancer, skin cancer, lung cancer, colon cancer, prostate cancer, and lymphoma. Symptoms vary depending on the type and strength. Immunity can natural transition that club of biomedical changes that seen in human body progress [1]. It can an early begin research topic that has been traditionally treated with force of attraction. Medically with a fairly low level of success and does not address the most significant result, which include depression, sign of fearness, poignant, linking and insomnia. Medicine has been shown in preliminary studies to address and treat specific symptoms of lupus. Some studies show a significant improvement in symptoms compared to that of family history; however these studies need to be reproduced on a larger scale to influence the medical community that has not familiar with therapy. 


\section{References}

1. Paranjape RS, Acharyulu GS, Krishnamurthy PV, Padma R. Cell mediated immunity in children with tuberculous meningitis. Indian Pediatr 123: 127-134 (1986).

2. Wallis RS, Paranjape R, Phillips M. Identification of Two Dimensional Gel Electrophoresis of a 58 Kilodalton TNF inducing protein of M. Tuberculosis Infection and Immunity (1993). 61(2), PP: 627-632.

3. Rahul Hajare. Can Otolaryngology Capture Window Cancer in Middle Adulthood? Ann Clin Lab Res 5: 205 (2017).
4. Kazemipour N, Qazizadeh H, Sepehrimanesh M, Salimi S. Biomarkers identified from serum proteomic analysis for the differential diagnosis of systemic lupus erythematosus. Lupus 24: 582-587 (2015).

5. Quinn TC, Brookmeyer R, Kline R, et al. Feasibility of Pooling Sera for HIV-1 Viral RNA to Diagnose Acute, Primary HIV-1 Infection and Estimate HIV-1 Incidence. AIDS 14: 2751-2757 (2000).

6. Rahul Hajare. The Aggression is an Early Cause of Cancer. A Narrative Review of Classical to Modern Scientific Literature. Chronicle Med Surg 1.2: 67-68 (2017). 